BACKGROUND: Different forms of chronic airway inflammation may involve diverse pathogenic elements. In general, deficient defence response is a feature of chronic obstructive pulmonary disease (COPD), whereas distorted immunoregulatory mechanisms lead to development of asthmatic symptoms. In addition to diverse effector mechanisms, the cellular and humoral elements participating in the development of immune response may appear to be different in COPD and bronchial asthma (BA) patients.

Aims: To evaluate the immunoregulatory properties of $T$ cells and monocytes in cultures of peripheral blood mononuclear cells (PBMC) and to determine the chosen cytokine profiles in COPD and BA patients.

Metbods: The microcultures of PBMC from COPD and BA patients were assessed for the $T$-cell response to mitogens, saturation of interleukin (IL)-2 receptors, T-cell suppressive activity and monokine influence on lymphocyte proliferation. Concomitantly, the cytokine (IL-1 $\beta$, interleukin-1 receptor antagonist, tumour necrosis factor- $\alpha$, IL-4, IL-6, IL-8) concentrations were determined in the serum, the broncho-alveolar lavage fluid and in the culture supernatants.

Results: The T-lymphocyte reactions (response to phytohaemagglutinin, IL-2 receptor saturation, suppressive activity) were lower in $\mathrm{BA}$ pateints than in COPD patients. Reversely, the immunogenic activity of monocytes (IL-1 $\beta$ versus IL-1ra production) was higher in BA patients than in COPD patients. The highest values of cytokine concentrations were found in the culture supernatants. The concentrations of tumour necrosis factor- $\alpha$, IL- 4, IL-6 and IL-8 were significantly higher and the concentration of IL-1ra was lower in BA patients than in COPD patients.

Conclusion: The assessments of cellular immunoregulatory properties and cytokine profiles in the cultures of blood mononuclear cells may prove helpful for diagnostic and therapeutic discrimination between BA and COPD patients.

Key words: Asthma, Chronic obstructive pulmonary disease, Immunopathogenesis, Cell-mediated immunoregulatory mechanisms, Cytokine profiles

\section{Cellular and cytokine immunoregulation in patients with chronic obstructive pulmonary disease and bronchial asthma}

\author{
Wanda Stankiewicz ${ }^{1}$, Marek P. Dạbrowski ${ }^{1, C A}$, \\ Andrzej Chcialowski and Tadeusz Plusa ${ }^{2}$
}

${ }^{1}$ Military Institute of Hygiene and Epidemiology, Department of Microwave Safety, Immunology Laboratory and ${ }^{2}$ Central Clinical Hospital of Military School of Mecicine, Clinic of Pneumonology and Allergy, 00909 Warsaw, Szaserów 128, Poland

\author{
${ }^{\mathrm{CA}}$ Corresponding Author \\ Tel: +48226816134 \\ Fax: +48 228104391 \\ E-mail: wanda_stankiewicz@poczta.onet.pl
}

\section{Introduction}

Bronchial asthma (BA) and chronic obstructive pulmonary disease (COPD) have become an increasing problem for contemporary medicine and public health. Epidemiologic data suggest that the prevalence of bronchial hyperreactivity observed in patients with BA and COPD has increasing frequency. Identification of factors and mechanisms that have caused these increases may contribute for invention of a new preventive strategy to control the inflammatory process in the airways.
Chronic airway inflammation may develop along different pathogenic pathways responsible for incidental recurrent bronchial constriction and clinical appearance of COPD or BA. There are some wellestablished differences between the repertoires of cellular and humoral elements involved in both processes. ${ }^{1-9}$ TCD8 lymphocytes, neutrophils, macrophages, leukotriene LTB 4 , tumour necrosis factor- $\alpha$ (TNF- $\alpha$ ) and interleukin (IL)-8 are the main representatives of the local inflammatory milieau of COPD, whereas mast cells, basophils, active eosinophils, macrophages, TCD 4 cells of $\mathrm{T}$ helper $(\mathrm{Th})_{2}$ type, 
immunoglobulin (Ig)E, LTB4, histamine, IL-4, IL-5, IL-6 and IL-13 comprise the immune inflammatory elements characteristic of BA. In contrast to the number of participating elements, both pathways of immune response do not result in a final resolution, but contribute to further progression of the inflammatory state, thus representing profound, although different in nature, sorts of immune disabilities. If, in general, deficient defence mechanisms can be estimated as a pathogenic cause of progression of COPD, the main reasons for occurence of $\mathrm{BA}$ generate rather from distorted immunoregulatory properties of the immune system.

To gain an immunodiagnostic insight that could help to discern between COPD and BA patients who demonstrate exacerbated bronchospastic symptoms, we have assessed their blood-derived peripheral blood mononuclear cells (PBMC) for immunoregulatory properties [lymphocyte responses to phytohaemagglutinin (PHA) and to concanavalin A (Con A), Con A-induced T-cell suppressive activity (SAT index), monokine (interleukin-1 receptor antagonist/IL-1 $\beta$ ) influence on lymphocyte proliferation (LM index), and saturation of lymphocyte IL-2 receptors (IL-2 index)], which were tested in the microculture system. ${ }^{10}$ Concomitantly, the serum, the broncho-alveolar lavage (BAL) fluid and the supernatants of cultured PBMC were assessed for concentrations of the chosen cytokines (TNF- $\alpha$, IL-1 $\beta$, IL-1 ra, IL- 4 , IL-6 and IL-8) to compare their quantities and mutual proportions depending on the source of the tested samples and on their origin from COPD or BA patients.

The choice of such a repertoire of immunological tests was based on the following predictions.

1. The mononuclear cell population isolated from peripheral blood contains both $\mathrm{T}$ cells and monocytes (antigen-presenting cells) in quantities and with typical functional immunoregulatory properties characteristic for the donor. These cells, set up in the culture and tested under appropriate experimental conditions, ${ }^{10,11}$ may deliver in vitro informations on the actual functional state of immune cells existing in vivo.

2. The type and the concentration of elaborated cytokines also characterise the functional state of immune cells. The respective cytokines can be found in vitro in close vicinity to the responding cells (in the culture supernatants) or in vivo at the more distant humoral peripheries from the site of their production (e.g. in the BAL fluid or in the blood serum). In this respect, testing sites in the possibly closest humoral vicinity can be expected to yield the most accurate information on the actual functional state of a cellular sources of assessed cytokines. For this reason, the assessments of chosen cytokines were performed concomitantly in the serum and in the BAL fluid as well as in the supernatants of cultured PBMC of each patient.

3. If the results of the tests performed in the cultures of PBMC appear to be comparable with the results of cytokine analysis in the BAL fluid, and therefore sufficient for diagnostic discrimination between COPD and BA patients, the performance of BAL could be abandoned, in some cases at least, for the favour of PBMC assessments that are obviously much less harmful for the patient.

\section{Materials and methods}

\section{Patients}

The investigations were carried out in the group of 20 patients with COPD (12 men and eight women; age range, 38-50 years) and in the comparable group of 20 patients with BA (11 men and nine women; age range, 32-45 years) who demonstrated mild exacerbation of bronchospastic symptoms. They did not receive any systemic or inhaled corticosteroids for at least 3 months before admission to the study. The diagnosis of COPD or BA was established on the grounds of a routine subjective and objective clinical examination, and was confirmed by the appearance of atopic symptoms (positive skin-prick tests to common dust allergens, elevated level of total serum IgE) in BA patients and by the lack of such symptoms in COPD patients. They all expressed conscious consent for participation in the study and the investigations have received approval from the Ethical Commission of the Central Clinical Hospital of Military School of Medicine in Warsaw. Before administration of the therapy, the patients were tested immunologically and BAL was performed in all of them.

\section{Immunological tests}

\section{PBMC microcultures}

PBMC were isolated by density gradient sedimentation $(1.007 \mathrm{~g} / \mathrm{ml}$; Ficoll-Paque Pharmacia LKB Uppsala, Sweden) of heparinised venous blood samples taken from the patients. After determination of the cell viability, the microcultures were set up in triplicate in Linbro (Flow-Laboratories, Irvine, Scotland) round-bottomed, 96-well microplates, as described previously. ${ }^{10}$ In brief, the cultures, each containing $10^{5}$ cells in $0.2 \mathrm{ml}$ of RPMI 1640 supplemented with $15 \%$ of inactivated autologous serum, were incubated at $37^{\circ} \mathrm{C}$ in an ASSAB incubator (Uppsala, Sweden) in a humified atmosphere of $5 \% \mathrm{CO}_{2}$ in air for $72 \mathrm{~h}$. Respective triplicates of microcultures were left without stimulation or were stimulated with PHA (HA16, $0.4 \mu \mathrm{g} /$ culture, optimal dose; Murex Biotech Ltd., Dartford, UK) or Con A ( $8 \mu \mathrm{g} /$ culture; Sigma, St Louis, MO, USA). At $24 \mathrm{~h}$, some triplicates of microcultures were re-arranged as described elsewhere. ${ }^{10,11}$ For the last $18 \mathrm{~h}$ of incubation, the 
Table 1. Response to mitogens [PHA and Con $\left.A\left(\mathrm{dpm} \times 10^{3} / \mathrm{culture}\right)\right]$ and immunoregulatory indices (SAT index, LM index, and IL-2 receptor saturation) in PBMC microcultures from patients with COPD and BA

\begin{tabular}{|c|c|c|c|c|c|c|}
\hline \multirow[t]{2}{*}{ Patients } & \multicolumn{6}{|c|}{ PBMC microcultures } \\
\hline & $\begin{array}{c}\text { Spontaneous } \\
\text { 3HTdR } \\
\text { incorporation }\end{array}$ & PHA & Con A & $\begin{array}{l}\text { SAT } \\
\text { index }\end{array}$ & $\begin{array}{c}\text { LM } \\
\text { index }\end{array}$ & $\begin{array}{c}\mathrm{IL}-2 \\
\text { receptor } \\
\text { saturation (\%) }\end{array}$ \\
\hline $\begin{array}{l}\text { COPD }(n=20) \\
\text { BA }(n=20) \\
\text { Statistical significance }\end{array}$ & $\begin{array}{l}1.4 \pm 0.3 \\
1.6 \pm 0.4 \\
\text { N.S. }\end{array}$ & $\begin{array}{c}67.6 \pm 10.2 \\
49.5 \pm 11.1 \\
<0.01\end{array}$ & $\begin{array}{l}31.5 \pm 4.5 \\
32.7 \pm 6.8 \\
\text { N.S. }\end{array}$ & $\begin{array}{c}22.1 \pm 5.2 \\
15.2 \pm 3.6 \\
<0.01\end{array}$ & $\begin{aligned} 8.3 & \pm 4.1 \\
12.1 & \pm 6.2 \\
& <0.01\end{aligned}$ & $\begin{array}{l}84 \pm 15 \\
73 \pm 26 \\
<0.02\end{array}$ \\
\hline
\end{tabular}

Data presented as mean \pm standard deviation. N.S., not significant.

microcultures were added with $\left[{ }^{3} \mathrm{H}\right]$-thymidine (3HTdR) (specific activity, $5 \mathrm{C} / \mathrm{mM}$; Amersham, Chalfont, UK) in a dose of $0.4 \mu \mathrm{C} /$ culture. After harvesting the cultures, the measurements of incorporated radioactivity were carried out in a Packard Tri-carb 2100 TR liquid scintillation analyser (Packard Instrument Company, Meriden, USA) and the results were expressed as a mean value of disintegrations per minute (dpm) (standard deviation for each triplicate. The following parameters of T-lymphocyte and monocyte functions were assessed: (a) spontaneous $3 \mathrm{HTdR}$ incorporation, (b) T-cell response to PHA, (c) T-cell response to Con A, (d) the SAT index, (e) the IL-2 index, and (f) the LM index.

\section{Cytokine assessments}

The samples of the blood serum, the BAL fluid and the PBMC culture supernatants were collected, frozen $\left(-70^{\circ} \mathrm{C}\right)$ and subsequently tested by enzyme-linked immunosorbent assay in the universal microplate analyser Elx800 (Biokom (Warsaw-Janki), Poland) for the presence of TNF- $\alpha$, IL-1 $\beta$, IL-1 ra, IL- 4 , IL- 6 and IL-8 with the use of respective Quantikine kits (R\&D, Abingdon, UK). The results were expressed as a concentration of respective cytokine $(\mathrm{pg} / \mathrm{ml})$ in the tested sample.

The BAL fluid was obtained in the same way in all the patients. Briefly, about $80 \mathrm{ml}$ of the fluid was retrieved from the BAL performed with the three portions (each of $50 \mathrm{ml}$ ) of $0.9 \% \mathrm{NaCl}$ given consecutively into the airways during the bronchoscopy. The retrieved volume of the BAL fluid was centrifuged and the part of obtained cell-free supernatant (about $3 \mathrm{ml}$ ) served for cytokine assessments. No detailed cytologic analysis in this part of BAL fluid has been performed because only scanty cells or cellular debris were present in the sediment. The PBMC microculture supernatants, in which the cytokine measurements were performed, were collected from the separate sets of non-stimulated, triplicate microcultures at $24 \mathrm{~h}$ of incubation.

The results of performed estimations in the PBMC cultures, the blood serum and the BAL fluid were compared between COPD and BA patients. The observed differences of the mean values were analysed using the Student $t$-test to estimate their statistical significance.

\section{Results}

\section{PBMC microcultures}

The viability of cells tested at the end of the cultures remained at the level of $80 \%$ of the initial number of living cells $\left(10^{5}\right)$ in the culture. The results of the tests performed in PBMC microcultures are presented in Table 1. The spontaneous incorporation of $3 \mathrm{HTdR}$ was low and similar in the PBMC cultures from COPD patients and from BA patients. In the response to PHA, the mononuclear cells of COPD patients proliferated more vigorously $\left[(67.6 \pm 10.2) \times 10^{3} \mathrm{dpm} /\right.$ culture] than the PBMC of BA patients $[(49.5 \pm 11.1)$ $\times 10^{3} \mathrm{dpm} /$ culture], and the difference was statistically significant $(p<0.01)$. The cells of both groups of patients responded well to Con A stimulation and no significant differences were observed between them. In contrast to that, the Con A-induced suppressive activity of $\mathrm{T}$ cells (the SAT index) was significantly lower $(p<0.01)$ in BA patients $(15.2 \pm$ $3.6)$ than in COPD patients $(22.1 \pm 5.2)$.

The LM index, which represents the competitive influence of IL-1ra and IL-1 $\beta$ on the lymphocyte proliferation (see also Table 2) was significantly lower $(p<0.01)$ in the PBMC cultures from COPD patients $(8.3 \pm 4.1)$ than in the respective cultures from $\mathrm{BA}$ patients $(12.1 \pm 6.2)$. The saturation of IL-2 lymphocyte receptors was higher in the COPD group ( $84 \pm$ $15 \%)$ than in the BA group of patients $(73 \pm 26 \%)$, and the observed difference was statistically significant $(p$ $<0.02$ ).

\section{Cytokine assessments}

The cytokine concentrations found in the blood serum, the BAL fluid and in the supernatants of PBMC cultures of COPD and of BA patients are presented in Table 2 . 
Table 2. Concentrations $(\mathrm{pg} / \mathrm{ml})$ of chosen cytokines in the serum, the BAL fluid and in the supernatants (SN) of PBMC cultures from COPD and BA patients

\begin{tabular}{|c|c|c|c|c|c|c|}
\hline \multirow[t]{2}{*}{ Cytokine } & \multicolumn{3}{|c|}{ COPD patients $(n=20)$} & \multicolumn{3}{|c|}{ BA patients $(n=20)$} \\
\hline & Serum & BAL fluid & SN & Serum & BAL fluid & SN \\
\hline $\mathrm{TNF}_{-\alpha} \alpha$ & $16.0 \pm 7.7^{\mathrm{a}}$ & $20.7 \pm 3.5$ & $141.0 \pm 32.1$ & $17.5 \pm 6.1$ & $13.1 \pm 3.7^{*}$ & $169.0 \pm 48.6^{*}$ \\
\hline $\mathrm{IL}-1 \beta$ & $15.3 \pm 0.9$ & $74.4 \pm 11.0$ & $950.0 \pm 86.2$ & $13.2 \pm 1.7$ & $59.4 \pm 15.2^{*}$ & $1020.0 \pm 191.2$ \\
\hline IL-1ra & $449.0 \pm 51.7$ & $2172.0 \pm 200.8$ & $4230.0 \pm 433.1$ & $360.2 \pm 64.8^{*}$ & $2290.0 \pm 181.0$ & $2800.0 \pm 680.4^{*}$ \\
\hline IL-4 & $20.9 \pm 1.4$ & $276.5 \pm 7.3$ & $79.0 \pm 18.3$ & $17.2 \pm 1.1^{*}$ & $233.6 \pm 17.2^{*}$ & $96.0 \pm 26.3^{*}$ \\
\hline IL-6 & $45.6 \pm 3.3$ & $423.2 \pm 38.0$ & $1392.0 \pm 206.4$ & $48.6 \pm 5.6$ & $405.0 \pm 59.4$ & $2600.0 \pm 708.2 *$ \\
\hline IL-8 & $25.9 \pm 2.5$ & $148.3 \pm 13.0$ & $229.0 \pm 26.8$ & $19.1 \pm 3.8^{*}$ & $164.4 \pm 11.3$ & $321.0 \pm 38.5^{*}$ \\
\hline
\end{tabular}

Data presented as mean \pm standard deviation, except a mean \pm SEM.

* Statistically significant differences $(p<0.01)$ between COPD and BA patients for the serum, the BAL fluid and the SN of the PBMC culture, respectively.

\section{Serum samples}

The concentrations of cytokines detected in the serum were low, with the exception of IL-1 ra, and the values of IL-1 ra, IL- 4 and IL- 6 found in BA patients were lower than in COPD patients. The observed differences were statistically significant $(p<0.01)$.

\section{BAL fluid}

The concentrations of cytokines present in the samples of BAL fluid of the both groups of patients were distinctly higher than the respective cytokine concentrations found in the serum. The exception was TNF- $\alpha$, whose concentration in the BAL fluid of BA patients was lower than in the serum of these patients. The concentrations of TNF- $\alpha$, IL- $1 \beta$ and IL- 4 were significantly lower in BA patients than in COPD patients, but the ratio of IL-1 ra/IL-1 $\beta$ concentration was higher in BA patients $(38.5 \pm 7.4)$ than in COPD patients $(29.2 \pm 3.8)$. The observed differences were statistically significant $(p<0.01)$.

\section{Supernatants of PBMC cultures}

The concentrations of TNF- $\alpha$, IL-1 $\beta$, IL-1 ra, IL-6 and IL8 found in the supernatants of PBMC cultures exceeded considerably the respective values found in the serum or in the BAL fluid of the both groups of patients. In contrast, the concentration of IL- 4 was higher than in the serum but lower than in the BAL fluid. The concentration of IL-4 found in BA patients was significantly higher than that in COPD patients. Also, the concentrations of TNF- $\alpha$, IL- 6 and IL-8 were significantly higher in BA patients than in COPD patients. No differences were observed between the concentrations of IL-1 $\beta$ in BA and COPD patients but the value of IL-ra was significantly lower in BA patients and, therefore, the ratio of $\mathrm{IL}-1 \mathrm{ra} / \mathrm{IL}-1 \beta$ concentration also was lower in BA patients than in COPD patients $(2.7 \pm 0.6$ versus $4.4 \pm 0.4$, respectively).
The values of cytokine concentrations found in the serum, the BAL fluid and the culture supernatants could not be compared directly because of the different sources of the sample origin and the different methods of preparation. Nevertheless, the samples of the same origin, which represented the highest values of cytokine concentration and the most evident differences between the groups of patients, could be estimated as particularly valuable for diagnostic purposes.

\section{Discussion}

The elevated total serum IgE, the presence of IgE specific to common dust allergens and positive results of skin-prick tests to the air distributed allergens (house-dust mites, cockroaches, pets and the fungi alternaria) are the main diagnostic features that discriminate the atopic asthma from the COPD in patients demonstrating incidental symptoms of bronchial constriction. ${ }^{8,12-15}$

The enlisted features represent the final efectory elements that generate from a long chain of preceding immunopathogenic events responsible for development of a chronic inflammatory state in the airways. Recognition of the nature and the sequence of these events may be helpful not only for the settlement of a discriminative clinical diagnosis, especially in the cases with less evident final allergic symptoms, but also for a better understanding of the mechanisms that lead to the airway inflammation.

Between the pathway of the $\mathrm{Th}_{2}$-mediated mechanism responsible for allergic inflammation and the $\mathrm{Th}_{1}$ mechanism preponderant in a non-allergic inflammatory reaction, both qualitative and quantitative differences can be found. They relate to the profile of cytokines involved and to the actual functional state of immunoregulatory cells, including T-cell suppressive activity and production by monocytes of proinflammatory (IL-1 $\beta$ ) and anti-inflammatory (IL-1ra) monokines. To gain an immunodiagnostic insight into these elements, several types of the clinical material 
can be sampled. The results of our investigations seem to suggest that, among different samples, including blood serum and BAL fluid, the mononuclear cells isolated from the blood and cultured under appropriate experimental conditions may deliver the most accurate and comprehensive informations on the immune mechanisms involved in the development of airway inflammation. Moreover, the vein puncture is much more convenient for the patients than the traumatic procedure of performed BAL.

The results of our in vitro assessments of the functional properties of PBMC found in BA patients and in COPD patients are in line with the present conception of a different immune background accounting for development of allergic or non-allergic forms of airway inflammation.

In BA patients, the parameters representing the T-lymphocyte immunocompetence and immunoregulatory abilities of $\mathrm{a} \mathrm{Th}_{1}$ nature (response to PHA, Con A-induced suppressive activity and IL-2 receptor saturation) expressed the values that were significantly lower than the respective values found in COPD patients (Table 1 ).

The values of the LM index, which represent, in a reciprocal manner, a competitive influence of IL-1 ra and IL-1 $\beta$ on the lymphocyte proliferative response, ${ }^{11}$ were significantly higher in BA patients than in COPD patients. The results show the existence of a relative preponderance of a pro-inflammatory activity of IL-1 $\beta$ over an anti-inflammatory activity of IL-1ra in BA patients. The absolute value of the IL-1ra concentration found in the supernatants of PBMC cultures of BA patients was considerably lower $(2800.0 \pm 680.4 \mathrm{pg} /$ $\mathrm{ml})$ than the respective value found in COPD patients $(4230.0 \pm 433.1 \mathrm{pg} / \mathrm{ml})$, whereas the concentrations of IL-1 $\beta$ were similar in the both groups of patients (Table 2 ). On the contrary, the values of IL-1 $\beta$ and IL1ra concentrations found in the culture supernatants in healthy volunteers, and presented in our earlier publication $^{11}$ were $170 \pm 90$ and $1900 \pm 810 \mathrm{pg} / \mathrm{ml}$, respectively, which produces a relatively high IL-1 ra/ IL-1 $\beta$ ratio $(11.1 \pm 7.5)$ and a low value of the $\mathrm{LM}$ index $(8.2 \pm 2.5)$.

The assessments of the IL-1ra/IL-1 $\beta$ concentration ratio within the humoral environment of responding cells may provide a diagnostic insight into the progression of the ongoing immune response and into the actual stage of the development of inflammatory process. In general, low values of the IL-1ra/IL-1 $\beta$ ratio characterise the progressive phase whereas high values mark the regressive phase of both processes. ${ }^{11,16}$ The comparison of the observed values of the LM index suggests that the airway inflammation was more intense in BA patients than in COPD patients.

The assessments of the other cytokines in the supernatants of PBMC cultures of BA and COPD patients revealed the existence of differences in the concentrations of $\mathrm{TNF}_{-} \alpha$, IL- 4 , IL-6 and IL-8. The concentrations of these cytokines were considerably higher in BA patients than in COPD patients. Interestingly, the serum values of TNF- $\alpha$, IL- 4 , IL- 6 and IL-8 found in COPD and BA patients remained, on average, at the level accepted for healthy volunteers (15.6, $31.2,12.5$ and 31.2, respectively, cited after the R\&D Quantikine kits producer). In contrast to that, the values found in our experiments in the culture supernatants have differed considerably from the values described by the cytokine kits producer as a characteristic for the blood mononuclear cell culture supernatants in apparently healthy volunteers. The observed differences exceeded significantly the minimal cytokine concentrations guaranted by $R \& D$ as detectable (e.g. $<4.4 \mathrm{pg} / \mathrm{ml}$ for TNF- $\alpha, 14 \mathrm{pg} / \mathrm{ml}$ for IL$1 \mathrm{ra}, 0.7 \mathrm{pg} / \mathrm{ml}$ for IL-6, and $10 \mathrm{pg} / \mathrm{ml}$ for IL-8).

The present observations confirm the existence of pathogenic imbalance between the pro-inflammatory activities of IL-1 $\beta$ and the diminished anti-inflammatory potential of IL-1ra, which could be expected in BA patients. The IL-1 ra/IL-1 $\beta$ concentration ratio was higher in COPD patients $(4.4 \pm 0.4)$ than in $\mathrm{BA}$ patients $(2.7 \pm 0.6)$, and the difference was statistically significant $(p<0.01)$. In these circumstances, the elevated concentrations of TNF- $\alpha$, IL-4, IL-6 and IL8 could result from the increased activity of IL- $1 \beta$, which strongly stimulates the production of numerous pro-inflammatory agents, including prostaglandins, cytokines and chemokines. ${ }^{16-18}$

Two cytokines, IL- 4 and IL-5, which belong to the $\mathrm{Th}_{2}$ pathway of the immune response, play an essential role in the development of allergic inflammation. IL-5 induces terminal differentiation of eosinophils and contributes to the airway eosinophilia, ${ }^{19,20}$ whereas IL- 4 stimulates the proliferation of B lymphocytes, the production of immunoglobulins and induces the switch of the produced immunoglobulins from IgM to IgE class. ${ }^{21,22}$ The assessments of the one of these cytokines were included in our study and the results show that the concentrations of IL- 4 found in the supernatants of PBMC cultures were significantly higher in BA patients than in COPD patients. The fact that the concentrations of IL- 4 found in the supernatants of PBMC cultures was lower than in the BAL fluid in both groups of patients could be attributed to the procedure of PBMC isolation from the vein blood. During this procedure, the mast cells and the basophils, which are important but not exclusive producers of IL-4, could be lost on the density gradient.

In conclusion, the results of the present investigations indicate that in addition to the routine allergic tests that discriminate between BA and COPD in patients with the symptoms of bronchial constriction, the assessments of PBMC performed in the culture may deliver important information on the nature and the progression of the airway inflammation. This 
information, comprising the cell-mediated immunoregulatory properties of the immune system and the cytokine profile assessed in the culture supernatants, may prove to be helpful for discrimination between the allergic and non-allergic inflammation, and may contribute to the development of a new preventive and therapeutic strategy to control the course of inflammation in the airway.

\section{References}

1. Saetta M. Airway pathology of COPD compared with asthma. Eur Respir Rev 1997; 7: 211-215.

2. Jeffery PK. Bronchial biopsies and airway inflammation. Eur Respir J 1996; 9: 1583-1587.

3. Brown PH, Crompton GH, Greening AP. Proinflammatory cytokines in asthma. Lancet 1991; 338: 590-593.

4. Sad S, Marcotte R, Mosmann TR. Cytokine-induced differentiation of precursor mouse CD8+ T cells into cytotoxic CD8+ T cells secreting Th 1 or Th2 cytokines. Immunity 1995; 2: 271-279.

5. Walker C, Bauer W, Braun RK, et al. Activated T cells and cytokines in bronchoalveolar lavages from patients with various lung diseases associated with eosinophilia. Am J Respir Crit Care Med 1994; 150: 1038-1048.

6. Robinson DS, Hamid Q, Ying S, et al. Predominant Th2-type bronchoalveolar T-lymphocyte population in atopic asthma. N Engl J Med 1992; 326: 298-304.

7. Prescott S, Macaubas C, Holt BJ, et al. Transplacental priming of the human immune system to environmental allergens: universal skewing of initial T cell responses toward the Th2 cytokine profile. J Immunol 1998 ; 160: $4730-4737$.

8. Holt PG, Macaubas C, Strumbles PA, Sly PD. The role of allergy in the development of asthma. Nature 1999; 402 (Suppl): B12-B17.

9. Constant SL, Bottomly K. Induction of Th1 and Th2 CD4+ T cell responses: the alternative approaches. Annu Rev Immunol 1997; 15: 297-322.
10. Dabrowski MP, Dabrowska-Bernstein BK, Stasiak A, Gajkowski K, Korniluk S. Immunologic and clinical evaluation of multiple sclerosis patients treated with corticosteroids and/or calf thymic hormones. Ann NY Acad Sci 1987; 496: 697-706.

11. Dabrowski MP, Stankiewicz W, Szmigielski S. The level of lymphocyte response to delayed stimulation with PHA reflects the monocyte production of IL-1 and IL-1ra. XIV European Immunology Meeting EFIS [abstract 686]. Immunol Lett 2000; 73: 242.

12. Burrows B, Martinez FD, Halonen M, Barbee RA, Cline MG. Associacion of asthma with serum IgE levels and skin-test reactivity to allergens. $N$ Engl J Med 1989; 320: 271-277.

13. Varner AE, Lemanske RF Jr. The early and late response to allergen. In: Busse WW, Holgate ST, eds. Asthma and rhinitis. London: Blackwell Science, 2000: 1172-1185.

14. Busse WW, Lemanske RF Jr. Asthma. Advances in immunology. $N$ Engl J Med 2001; 344: 350-362.

15. O'Hollaren MT, Yunginger JW, Offard KP, et al. Exposure to an aeroallergen as a possible precipitating factor in respiratory arrest in young patients with asthma. $N$ Engl J Med 1991; 324: 359-363.

16. Dinarello CA. The role of interleukin-1-receptor antagonist inblocking inflammation mediated by interleukin-1. N Engl J Med 2000; 343: $732-734$.

17. Oppenheim JJ, Kovacs EJ, Matsushima K, Durum SK. There is more than one interleukin-1. Immunol Today 1986; 7: 45-56.

18. Dinarello CA, Wolff MS. The role of interleukin-1 in disease. $N$ Engl J Med 1993; 328: 106-113.

19. Sanderson CJ. Interleukin-5, eosiniphils, and disease. Blood 1992; 79: 3101-3109.

20. Shi H-Z, Xiao C-Q, Zhong D, et al. Effect of inhaled interleukin-5 on airway hyperreactivity and eosinophilia in asthmatics. Am J Resp Crit Care Med 1998; 157: 204-209.

21. Mire-Sluis AR, Thorpe R. Cytokines, San Diego, CA: Academic Press, 1998.

22. Wills-Karp M, Luyimbazi J, Xu X, et al. Interleukin-13: central mediator of allergic asthma. Science 1998; 282: 2258-2261.

Received 18 June 2002

Accepted 16 July 2002 


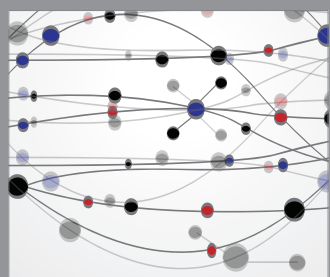

The Scientific World Journal
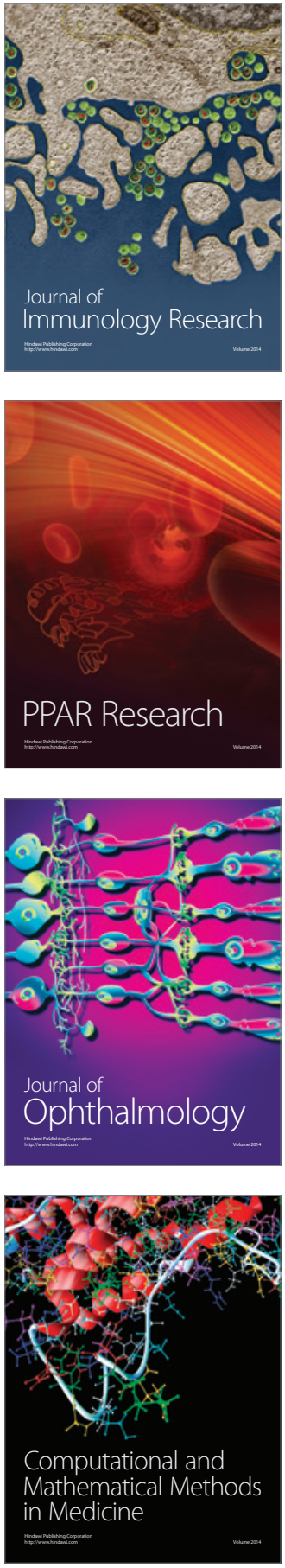

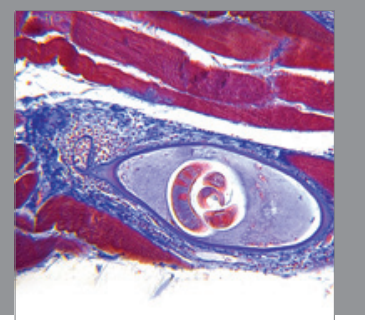

Gastroenterology

Research and Practice
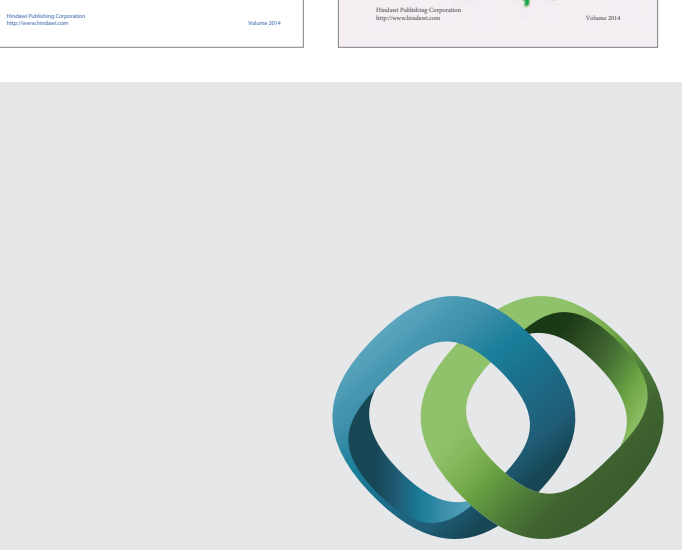

\section{Hindawi}

Submit your manuscripts at

http://www.hindawi.com
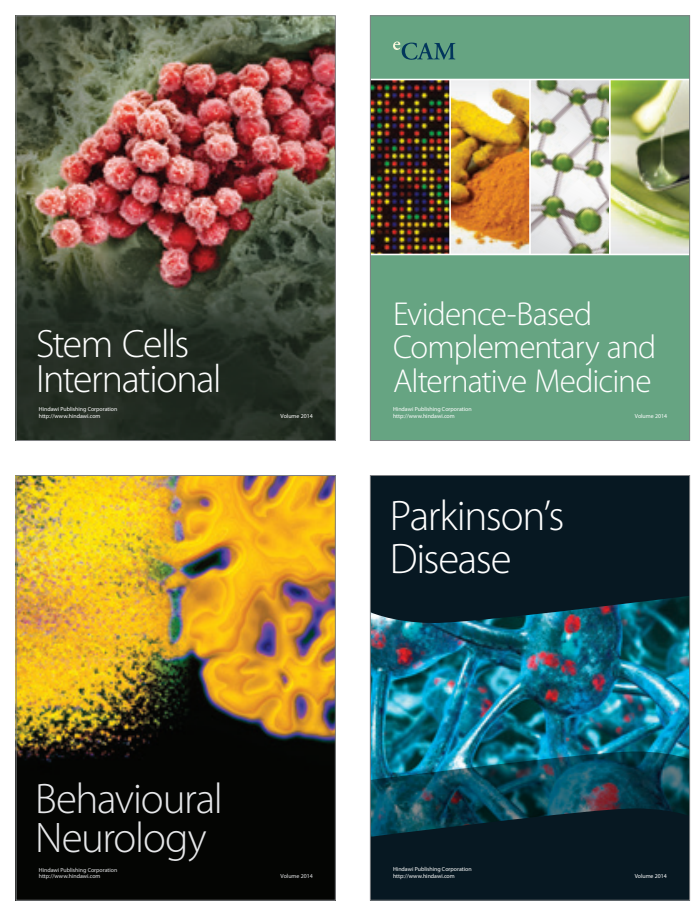

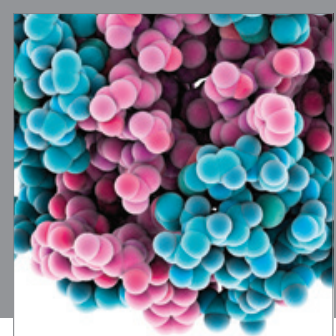

Journal of
Diabetes Research

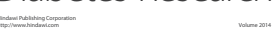

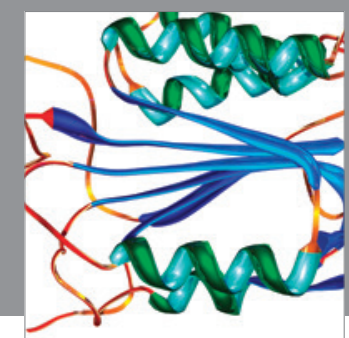

Disease Markers
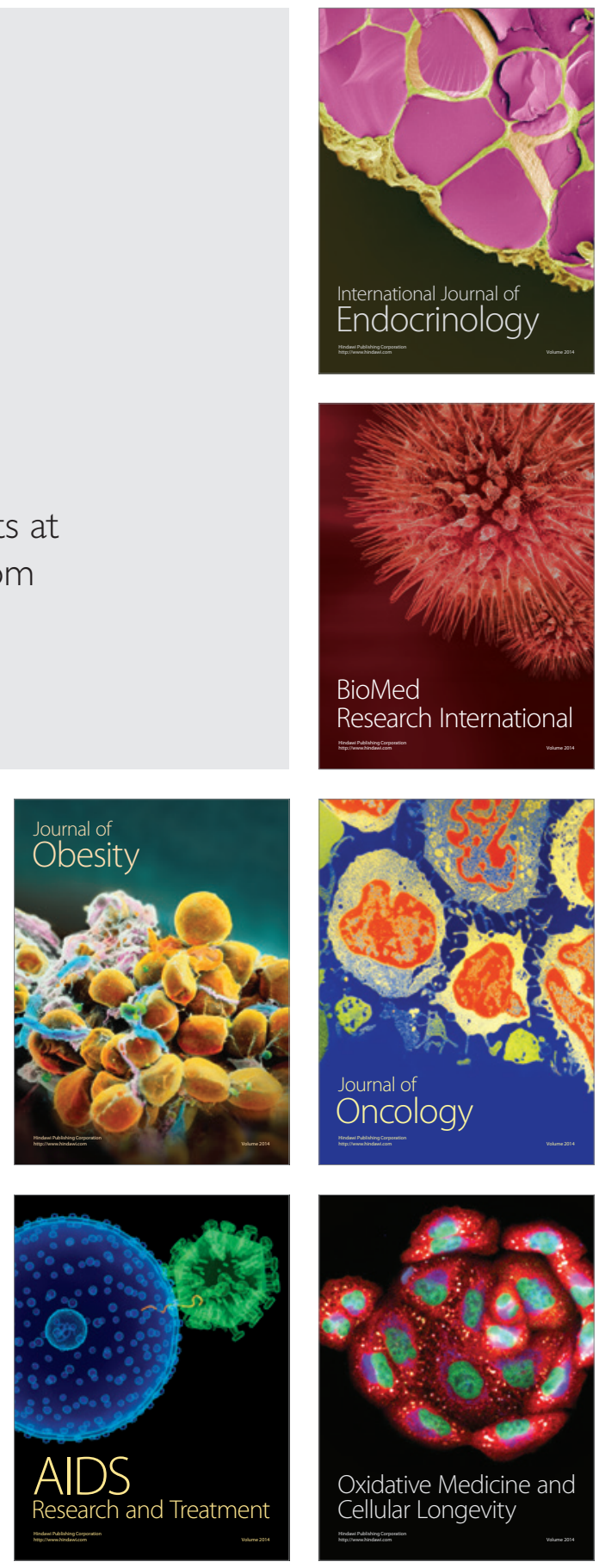